\title{
Acknowledgement:
}

This is a draft of a chapter that has been accepted for publication by Oxford University Press in the forthcoming book The Oxford Handbook of Emotional Development edited by Dr. Daniel Dukes, Prof. Andrea C. Samson, and Prof. Eric A. Walle, and due for publication in 2021. 
Developmental Methods for Emotion Dynamics

Jessica P. Lougheed

Purdue University

Author Note

Jessica P. Lougheed, Department of Psychology, University of British Columbia

Okanagan.

Correspondence concerning this article should be addressed to Jessica P. Lougheed, Department of Psychology, University of British Columbia Okanagan, 1147 Research Road, Kelowna, BC, V1V1V7. E-mail: Jessica.lougheed@ubc.ca 


\begin{abstract}
Emotions and their development are complex processes. Emotions are dynamic; involve multiple biological, psychological, and social systems; and can be idiosyncratic. However, much of the research on emotional development has used methods that do not capture the dynamic nature of emotions; focus only on one biological, psychological, or social system; and/or do not account for individual differences. I provide an overview of current methods for developmental studies on emotion dynamics. First, I introduce methods for examining emotions as dynamic processes. Then, I extend this discussion to multiple burst designs that capture emotion dynamics at multiple time scales (Ram \& Diehl, 2015). Throughout, I discuss approaches for both individual and interpersonal emotion dynamics that are applicable across the lifespan. I conclude with a discussion of future directions in the study of emotion dynamics and their development. Keywords: Emotion dynamics; emotion regulation; emotional development; research methods; developmental methods.
\end{abstract}


METHODS FOR EMOTION DYNAMICS

UNCORRECTED FIRST PROOF

Developmental Methods for Emotion Dynamics

Emotions and their development have long been conceptualized as dynamic processes that ebb and flow (Cole, Martin, \& Dennis, 2004; Thompson, 1994). Therefore, the study of emotion dynamics requires the use of methods that assess emotions across time and context. The study of emotion dynamics is burgeoning in part to innovations in technology and statistics that facilitate the uptake of dynamic approaches. However, dynamic approaches are still far from the norm in the field. In this chapter, I introduce developmental methods for examining emotion dynamics. I discuss methods for both individual and interpersonal processes, and the importance of the time scale (e.g., moments, hours, days, years) of unfolding dynamics. First, I discuss theoretical perspectives of time scales in emotion dynamics and their development. Then, I review methods for examining emotion dynamics at different time scales. I end with a discussion of future directions for developmental methods in emotion dynamics.

Several theoretical conceptualizations emphasize that emotions and their development are dynamic processes unfolding at multiple time scales (Hollenstein, Lichtwarck-Aschoff, \& Potworowski, 2013; Lewis, 2000; Lougheed, 2019). In terms of individual processes, Lewis (2000) conceptualized emotions as self-organizing at three time scales: micromomentary emotions at the smallest time scale, longer-lasting moods, and an enduring structure of personality. Lower-order system elements (i.e., momentary emotional states) coalesce into moods, which, through repetition, can form the bases of individual differences such as personality. These enduring personality structures in turn constrain the lower-order system elements of emotional states and moods, creating a self-organizing structure over development (Lewis, 2000). This perspective emphasizes that no conceptualization of emotional development is complete unless it accounts for processes that occur at more than one time scale. 
METHODS FOR EMOTION DYNAMICS

UNCORRECTED FIRST PROOF

In terms of interpersonal processes, Butler (2011) put forth a conceptualization of individuals in close relationships as temporal interpersonal emotion systems (TIES), in which each partner's emotion systems (physiological, behavioral, and subjective emotional responses) interact with each other's, creating a dyadic system in which emotions are regulated interpersonally over time. With a focus on the developmental dynamics of the parent-adolescent relationship, in my own work (Lougheed, 2019, 2020) I recast the dynamics of TIES within a multiple time scale framework to illustrate how momentary interpersonal dynamics coalesce into broader features of the relationship, which in turn constrain the momentary dynamics of TIES. These two perspectives emphasize that emotional development occurs in the context of relationships, which shape longer-term developmental processes. In turn, these developmental processes (e.g., features of the relationship) constrain how emotions are regulated moment-bymoment in the context of social interactions (see chapters MÉNDEZ LEAL \& SILVERS; RIEDIGER \& BELLINGTIER; and STEPHENS ET AL., for more on the social regulation of emotion, this volume).

Multiple time scale-perspectives on emotional development guide methods for examining the development of emotion. First, these perspectives provide guidance in defining the dynamic process of focus. Second, these perspectives are linked to methodological approaches that can help match developmental theory to method. Finally, these perspectives help move developmental science closer to uncovering the mechanisms of developmental change in emotion. In the following sections, I review research that highlights the importance of dynamic methods for the study of emotional development. I first discuss research methods for dynamics at short, moment-to-moment time scales. Then, I discuss methods for emotion dynamics at longer time scales across hours and days. I then discuss important future directions including the need to 
METHODS FOR EMOTION DYNAMICS

UNCORRECTED FIRST PROOF

incorporate multiple time scales into study designs to fully understand the mechanisms of developing emotion dynamics.

\section{Moment-to-Moment Dynamics}

To date, the most common approaches used to examine the development of emotions has been to observe individuals or dyads in emotion-eliciting tasks and have trained observers rate the occurrence of positive and negative emotion expressions using a microanalytic coding scheme (e.g., event-based or second-by-second). A few recent studies illustrate how innovative analytical methods can leverage data that have been microanalytically coded to capture individual and interpersonal emotion processes as they unfold over short time scales.

Individual dynamics. Several different analytical strategies have been used to examine temporal relations among individuals' emotion regulation strategy use and emotion expressions. The aim of studies using such approaches is often to examine whether or not the use of strategies is effective in down-regulating (i.e., resolving) negative emotions or up-regulating (i.e., enhancing) positive emotions. Building off of prior work that has used contingency analysis to examine changes in children's emotion expressions following the use of emotion regulation strategies (Buss \& Goldsmith, 1998), we recently used multilevel survival analysis (MSA) to estimate the timing of children's recurring anger expressions during a frustrating wait task from children's time-varying use of distraction and social bid strategies (Lougheed, Benson, Ram, \& Cole, 2019). MSA can be used to statistically test the timing of recurring behavioral events, and time-varying influences of that timing. We found that the likelihood of children's anger expressions increased in the moments when they used bidding strategies that kept their attention focused on a restricted item, but that the likelihood of anger expressions decreased in the moments when they used a distraction strategy. One advantage of this analytical approach is that 
METHODS FOR EMOTION DYNAMICS

UNCORRECTED FIRST PROOF

it allows inferences about the effectiveness of strategy use-if the use of behavioral strategies increases or decreases the likelihood of emotion expressions in time.

Dynamical systems approaches (Boker, 2001; Molenaar, 2004; Cole, Lougheed, Chow, \& Ram, 2020; Yang, Ram, Lougheed, Molenaar, \& Hollenstein, 2019) are also gaining momentum as methods for examining individual emotion dynamics. Statistical approaches using ordinary differential equations are one example, and this method can be used to examine temporal processes such as fluctuations, damping, and amplification of emotions (Boker, 2001). This method was used in one study to examine children's self-regulation during a frustrating wait task (Cole, Bendezú, Ram, \& Chow, 2017). It showed that 36-month-old children's use of emotion regulation strategies had a temporary damping effect on their negative emotion expressions, but this damping effect was not long-lived during the task. This modeling strategy enabled the authors to conclude that children's use of emotion regulation strategies at this age is far from mature.

Interpersonal dynamics. Increasing attention has been paid in recent years to the development of emotion dynamics in interpersonal contexts, spurred forth by theoretical conceptualizations of emotion regulation in the context of social interactions (Butler, 2011; Campos, Walle, Dahl, \& Main, 2011). Some studies have examined individuals as nested within dyads to examine the development of interpersonal emotion dynamics (e.g., Morris et al., 2011; see STEPHENS ET AL., this volume). Much of this work has examined physiological synchrony in parent-infant dyads, which points to the importance of biobehavioral synchrony in the early years to establishing the basis of an adaptive parent-child relationship and developing infant regulation (Feldman, 2007, 2012). Caregivers and infants begin to develop a rhythm of interaction that is reflected in synchrony at the physiological level through caregivers' behavioral 
METHODS FOR EMOTION DYNAMICS

UNCORRECTED FIRST PROOF

attunement to infant cues (Feldman, 2007; Fogel, 1993). The basis of the relationship is established through this process and infants learn the beginnings of physiological regulation.

Interpersonal synchrony has become a major topic in research on interpersonal emotion dynamics and has been studied in a variety of relationship types, including parent-child and romantic partners (see Harrist \& Waugh, 2002; Timmons, Margolin, \& Saxbe, 2015 for reviews). There are a number of methods for examining synchrony (Helm, Miller, Kahle, Troxel, \& Hastings, 2018). Versions of actor-partner interdependence models (APIMs; Kenny \& Kashy, 2011) including auto- and cross-lagged panel models allow researchers to test the influence of Partner A's emotions at time $t$ on Partner B's emotions at time $t+n$ (and vice versa), while controlling for the influence each partner's own emotions at the previous time point. This type of dynamic maps onto theoretical conceptualizations of synchrony and emotion transmission, in which one partner's emotions influence the other's at a subsequent time point (Butler, 2011). Across developmental periods, studies using these types of approaches have shown emotion transmission in parent-child dyads in infancy and childhood (Feldman, 2007) and adolescence (Papp, Pendry, \& Adam, 2009). For example, mother-adolescent dyads show greater cortisol synchrony when they are experiencing negative emotions (Papp et al., 2009). Emotion transmission has been shown across different domains of the emotion system, including sympathetic nervous system activity (Lougheed \& Hollenstein, 2018; Lougheed, Koval, \& Hollenstein, 2016), and expressed and experienced emotions (Mancini, Luebbe, \& Bell, 2016).

Innovative methods have also been used to examine temporal links between two individuals' observed behaviors. We used MSA to directly examine parental responses to children's and adolescent's expressed emotions from observations of behaviors during parentchild interactions (Lougheed, Craig, et al., 2016; Lougheed, Hollenstein, \& Lewis, 2016; 
METHODS FOR EMOTION DYNAMICS

UNCORRECTED FIRST PROOF

Lougheed, Hollenstein, Lichtwarck-Aschoff, \& Granic, 2015). Taking this approach showed that parents of children with externalizing problems tended to be less contingent in responding supportively to their children's negative emotion expressions than parents of typicallydeveloping children (Lougheed et al., 2015), and that a similar lack of contingent responses may also be related to adolescent internalizing symptoms (Lougheed, Craig, et al., 2016). Another study examining age differences in parent-adolescent dynamics used recurrence quantification analysis (Coco \& Dale, 2014) together with growth curve modeling to examine lead-lag associations in parent-adolescent emotion dynamics (Main, Paxton, \& Dale, 2016). This study showed that older adolescents supportively validate their mothers' emotions more than younger adolescents, but also that older adolescents tend more to be the "drivers" of negative emotional exchanges than younger adolescents.

A number of research studies have examined socioemotional flexibility of the dyadic parent-child system, which is the ability for a dyad to transition between emotions according to situational demands of the interpersonal interaction (Hollenstein et al., 2013). This type of flexibility is often examined by using microanalytically-coded observations of dyadic emotion expressions during one or more interaction context and deriving measures such as the number of observed transitions between dyadic emotion states, and the range of states expressed (Hollenstein, 2013). Developmental research on dyadic flexibility has shown that in early childhood, flexibility is negatively associated with the longitudinal development of children's externalizing behavioral problems (Hollenstein, Granic, Stoolmiller, \& Snyder, 2004; Lunkenheimer, Olson, Hollenstein, Sameroff, \& Winter, 2011). In middle childhood, children being treated for clinically-significant externalizing problems show increases in dyadic flexibility over the course of successful therapeutic treatment (Granic, O’Hara, Pepler, \& Lewis, 2007). 
METHODS FOR EMOTION DYNAMICS

UNCORRECTED FIRST PROOF

Flexibility increases during the adolescent transition before settling into a new, more established dynamic, which suggests a reorganization of parent-adolescent interaction dynamics at the microsocial level leading to longer-term developmental reorganization (Granic, Hollenstein, Dishion, \& Patterson, 2003). Flexibility in the parent-adolescent relationship is associated with both parental and adolescent psychosocial adjustment (i.e., lower internalizing symptoms; Lougheed \& Hollenstein, 2016; van der Giessen, Branje, Frijns, \& Meeus, 2013). Taken together, microanalytic observations of behaviors enable researchers to examine the processes of how emotions unfold both intra- and interpersonally. Researchers can examine important topics such as the time course of emotions, how emotions are affected by behavioral strategies, and how dyads respond to changing circumstances.

\section{Hourly and Day-to-Day Dynamics}

Emotion dynamics have also been examined at longer time scales during day-to-day life. The experience sampling method (ESM; Bolger \& Laurenceau, 2013) is the most common approach and enables researchers to prompt participants to respond to questionnaires throughout their day-to-day lives such as via participants' mobile phones. This approach captures emotion dynamics at a longer time scales than studies making use of behavioral observations, and the specific time scale depends on the number of samples per day. This approach allows researchers to examine individual and interpersonal emotion dynamics as they unfold in situ.

Individual dynamics. ESM has been used to investigate which emotion regulation strategies individuals tend to use in their daily lives, and which strategies tend to be more strongly associated with changes in emotional experiences (Brans, Koval, Verduyn, Lim, \& Kuppens, 2013). This approach has revealed valuable insights about the regulation of emotions in daily life, such as that among adults, distraction strategies tend to be the most commonly used, 
METHODS FOR EMOTION DYNAMICS

UNCORRECTED FIRST PROOF

that multiple strategies tend to be used in combination, and that rumination and suppression strategies are associated with decreases in positive and increases in negative emotional experiences (Brans et al., 2013). Studies using similar approaches in adolescent samples have also shown that rumination strategies tend not to be effective in resolving negative emotions, and are also associated with greater psychosocial adjustment difficulties (Silk, Steinberg, \& Morris, 2003).

The ESM method has yielded valuable information for developmental psychopathology. Greater depressive symptoms have been associated with greater emotional variability in day-today life in adolescence but not in late childhood (Larson, Raffaelli, Richards, Ham, \& Jewell, 1990), which suggests developmental changes in the associations between emotion dynamics and psychopathology. Emotion regulation dynamics also distinguish between youth experiencing clinically significant anxiety disorders from typically-developing youth (Tan et al., 2012). Specifically, adolescents experiencing anxiety do not differ from typically-developing adolescents in their responses to typical day-to-day experiences, but rather show heightened negative emotional reactivity to situations that are particularly emotionally challenging (Tan et al., 2012).

Interpersonal dynamics. In terms of interpersonal dynamics, ESM has been used most extensively in the context of adult romantic relationships (see Schoebi \& Randall, 2015 for a review; see also STEPHENS ET AL., this volume). When using ESM to collect data from individuals nested in relationships, it is common to prompt participants to respond to questionnaires at the same time. For example, by prompting participants to respond four times per day corresponding to personally-relevant times of day (e.g., before work, when reuniting with the partner at the end of the work day, before going to bed), researchers have examined the 
METHODS FOR EMOTION DYNAMICS

UNCORRECTED FIRST PROOF

extent to which individuals in couple relationships are influenced by each other's emotional experiences (Randall \& Schoebi, 2015). A variation of multilevel APIMs was used to statistically test if Partner A's emotional experiences predicted change in Partner B's subsequent emotional experiences (and vice versa). Greater susceptibility to partner's emotional experiences may be protective against future distress, potentially because this susceptibility may indicate greater opportunity for emotional co-regulation within the couple relationship (Randall \& Schoebi, 2015). Another study using this method showed that humor may be an effective strategy for interpersonal emotion regulation among romantic partners - a humorous remark by one partner may increase both partner's positive emotion and feelings of intimacy in the relationship (Horn, Samson, Debrot, \& Perrez, 2019).

One of the first examinations of parent-adolescent dynamics using ESM suggested that parents' and adolescents' emotional experiences are linked (Larson \& Richards, 1994). During periods when family members were at home, adolescent girls' emotional experiences predicted parental experiences, i.e., their emotions were "transmitted" to their parents at an hour-to-hour time scale (Larson \& Richards, 1994). More recently, a new analytic method called gridsequence analysis (Brinberg, Fosco, \& Ram, 2017; Brinberg, Ram, Hülür, Brick, \& Gerstorf, 2018) has been applied to ESM data of parent-adolescent daily reports of connectedness (feelings of closeness, trust, and support). This method converts categorical time series data into sequences, and then interdyad differences in those sequences (i.e., how measured behaviors unfold over time) can be examined. In one study, results showed interdyad differences in daily reports of parent-adolescent connectedness with some dyads showing stable, high connectedness over a few weeks and others showing variable connectedness and discrepant parent and adolescent reports (Brinberg et al., 2017). Taken together, ESM methods enable researchers to 
METHODS FOR EMOTION DYNAMICS

UNCORRECTED FIRST PROOF

examine intra- and interpersonal emotion dynamics in day-to-day life outside of the lab setting. Researchers can examine how individual's own use of emotion regulation strategies, or their partners', effect subsequent emotional experiences, and innovative analytic approaches facilitate the examination of complex patterns.

\section{Discussion and Future Directions}

Innovative methods are being used to examine emotion dynamics at different time scales, ranging from micro-momentary to hourly and daily. The most common approach for examining dynamics at the shortest time scales are recordings of behavioral expressions and physiological responses. The most common approach for examining dynamics at hourly and daily time scales is sampling individuals' experiences with ESM. Developmental science is seeing increasing uptake of such methods which is inspiring exciting new research directions. However, a comprehensive developmental picture of emotion dynamics will not be available until we combine innovative methods to assess dynamics across multiple time scales. The combination of intensive observations of behavior (short time scale) over longer periods of development (long time scale) are referred to as multiple burst designs (Ram \& Diehl, 2015). In this final section, I describe the few studies that have used multiple burst designs and point towards future directions using such methods.

Multiple time scale designs. In one of the first explications of development at multiple time scales, Nesselroade (1991) described short term change at the micro time scale (e.g., moments, seconds) as reflecting regulation and reinforcement processes, and changes at longer time scales (e.g., months, years) as resulting from developmental processes. Incorporating measurement "bursts" of intensive longitudinal data (micro time scale), repeatedly over developmental time (i.e., longitudinally over months or years), enables researchers to examine 
METHODS FOR EMOTION DYNAMICS

UNCORRECTED FIRST PROOF

how longer-term development emerges from momentary dynamics, and in turn how momentary dynamics are constrained by longer-lasting developmental structures (Nesselroade, 1991; Ram \& Diehl, 2015). The few studies to date that have used such multiple burst designs demonstrate its value to the study of developing emotion dynamics.

To examine developmental changes in micro time scale dynamics, multiple burst designs can involve repeating the same behavioral observation tasks at different ages (Cole, Lougheed, $\&$ Ram, 2018). Examples include repeated observations of mothers soothing infants during immunizations, a challenging emotional experience (Benson, Ram, \& Stifter, 2018; Stifter \& Rovine, 2015); young children during laboratory tasks that challenge their self-regulation (Helm, Ram, Cole, \& Chow, 2016; Morales et al., 2018); children and parents during structured and unstructured observations (Stoolmiller, 2016; Stoolmiller \& Snyder, 2014); and of parents and adolescents during conflict discussions (van der Giessen et al., 2013). It is critical to consider the potential for practice effects when using behavioral tasks repeatedly in multiple burst designs. The studies listed above are exemplars of designs that minimize this problem.

There are also a few examples that link dynamics at hourly and daily dynamics to developmental change, although they are less common. In one study, adolescents' emotion dynamics were examined with ESM over the course of treatment for major depressive disorder (Silk et al., 2011). Five bursts of experience sampling were conducted during an eight-week treatment protocol. Results showed that differences in the intensity and lability of negative emotional experiences between adolescents experiencing depression and typically-developing adolescents decreased during the course of treatment, which provides strong evidence that the effects of the treatment translated to the real-world context of daily emotional experiences (Silk et al., 2011). Another study shows how multiple burst designs can incorporate features of several 
METHODS FOR EMOTION DYNAMICS

UNCORRECTED FIRST PROOF

research methods (Ram et al., 2014). Research on lifespan development has the particular challenge of a "long" view of development spanning a broad range of ages. In this domain, longitudinal studies can potentially span generations of researchers to examine development over decades, which is not always feasible. An innovative approach combined cross-sectional measurement over a broad range of ages with longitudinal panel design, daily diary, and experience sampling protocols to capture age-related differences in daily emotion dynamics between the ages of 18 and 89 years old (Ram et al., 2014). Participants completed three 21-day measurement bursts and reported on emotional experiences and social interactions. Analyzing these data with multilevel models enabled the examination of how dynamics within bursts and individuals are related to age differences in emotions and social interactions (Ram et al., 2014).

Innovations in statistical analyses are facilitating the uptake of multiple time scale designs by making new methods accessible to developmental scientists. A multiple time scale multiphase latent basis growth model has recently been developed to allow the analysis of behavioral change at multiple levels: (1) within task, and (2) longitudinal changes in these within-task changes (Helm et al., 2016). The first application of this new method demonstrated how young children's self-regulatory behaviors change over the course of a frustrating laboratory task, and how these within-task changes become less fragmented over age in line with children's developing executive control (Helm et al., 2016). An extension of MSA enables researchers to model temporal contingencies between multiple streams of behaviors (i.e., dynamics at the micro time scale) within a broader structural equation model to examine how momentary dynamics mediate developmental processes over longer time periods (Stoolmiller \& Snyder, 2014). The first applications of this method showed how parent-child emotion dynamics are related to the development of children's antisocial behavior (Stoolmiller, 2016; Stoolmiller \& Snyder, 2014). 
METHODS FOR EMOTION DYNAMICS

UNCORRECTED FIRST PROOF

Another new method integrates differential equations, which can describe momentary non-linear dynamics, with multilevel growth modeling, which captures longitudinal change processes

(Benson et al., 2018). The first application of this method showed how caregiver-infant interactions can be partitioned into self-and co-regulatory processes, and longitudinal changes in those processes. It is clear that joint modeling approaches, which leverage combinations of statistical approaches (e.g., micro time scale approaches cast within multilevel or growth models), are the future of developmental methods for emotion dynamics.

Future directions. It is an exciting time for research on the development of emotion dynamics. New study designs and statistical approaches are enabling a greater matching of data analysis and hypothesis tests to complex developmental theories. At the same time, these new approaches are facilitating the elaboration and refinement of theoretical conceptualizations of emotion dynamics (Butler, 2011; Hollenstein et al., 2013; Lougheed, 2019). I propose several goals for developmental scientists as we move the study of emotion dynamics forward in new directions.

First, one goal is to explore and describe dynamics at the micro time scale. The analysis of micro time scale dynamics is relatively new in the history of emotion science. Research that focuses on exploring and describing emotion dynamics in different contexts (e.g., positive versus negative emotional contexts; with different interaction partners such as parents and peers; in different settings such as home versus school and work), at different ages, and among different groups (e.g., gender, family structure, ethnicity, socioeconomic status) are crucial for forming a foundation of knowledge on which to elaborate into multiple burst designs.

A second goal is to examine changes in short term dynamics longitudinally with multiple burst designs. Many developmental scientists may already have access to such data sets, as it has 
METHODS FOR EMOTION DYNAMICS

UNCORRECTED FIRST PROOF

been a common practice to conduct longitudinal studies using laboratory observations of behaviors. Now that statistical approaches for modeling dynamics at multiple time scales are becoming more accessible, researchers could conduct secondary analyses of data that are already available to accelerate the body of research on dynamics at multiple time scales. Such secondary data analyses will benefit the design of new studies of dynamics at multiple time scales as we develop and refine best practices for these designs.

Finally, mixed methods approaches will be a major asset to the study of developing emotion dynamics. For example, studies could employ multiple complementary methods such as behavioral observations at the micro time scale with approaches that capture hourly and daily dynamics in situ with ESM. This approach would enable the observation of changes in dynamics at three or more time scales and enable a detailed analysis of processes underlying developmental change in emotion dynamics (see Hollenstein \& Tsui, 2019 for more discussion of this type of design).

The uptake of new methods also warrants some cautions. For example, sufficient statistical power is a concern with all study designs and methods. As intensive longitudinal data, and the statistical approaches to analyze them, become more common, it is important for researchers to be well-trained in the more complex considerations for power (i.e., power at multiple levels, such as within-person/dyad and between-person/dyad; Bolger \& Laurenceau, 2013). Another concern involves the complexity of model specification — as a field we need to beware the dangers of contributing to a scientific body of work built on a foundation of model overspecification (e.g., inclusion of extraneous or too many predictors) and questionable research practices (e.g., $p$-hacking) which may be easier to intentionally or unintentionally employ with complex analyses where many analytic decisions must be made. 
METHODS FOR EMOTION DYNAMICS

UNCORRECTED FIRST PROOF

Conclusion. Development is nonlinear and proceeds at multiple time scales

(Nesselroade, 1991; Ram \& Diehl, 2015). The complexity of emotional development is what makes it such a rich area of study, but also what provides challenges to implementing research.

Between the increasing accessibility of advanced statistical methods, innovations in study designs, and theoretical emphases on dynamic processes, developmental scientists are wellpositioned to take giant leaps forward in understanding the complex processes at play in emotional development. 


\section{References}

Benson, L., Ram, N., \& Stifter, C. A. (2018). Using fishery models to examine self- and coregulation processes across multiple timescales. Structural Equation Modeling, 25(6), 906-923. doi: 10.1080/10705511.2018.1491313

Boker, S. M. (2001). Differential structural equation modeling of intraindividual variability. In L. M. Collins \& A. Sayer G. (Eds.), New methods for the analysis of change (pp. 5-27). Washington, DC: American Psychological Association.

Bolger, N., \& Laurenceau, J.-P. (2013). Intensive longitudinal methods: An introduction to diary and experience sampling research. New York, NY: Guilford Press.

Brans, K., Koval, P., Verduyn, P., Lim, Y. L., \& Kuppens, P. (2013). The regulation of negative and positive affect in daily life. Emotion, 13(5), 926-939. doi: 10.1037/a0032400

Brinberg, M., Fosco, G. M., \& Ram, N. (2017). Examining inter-family differences in intrafamily (parent-adolescent) dynamics using grid-sequence analysis. Journal of Family Psychology, 31(8), 994-1004. doi: 10.1037/fam0000371

Brinberg, M., Ram, N., Hülür, G., Brick, T. R., \& Gerstorf, D. (2018). Analyzing dyadic data using grid-sequence analysis: Interdyad differences in intradyad dynamics. The Journals of Gerontology Series B: Psychological Sciences and Social Sciences, 73(1), 5-18. doi: $10.1093 /$ geronb/gbw160

Buss, K. A., \& Goldsmith, H. H. (1998). Fear and anger regulation in infancy: Effects on the temporal dynamics of affective expression. Child Development, 69(2), 359-374. doi: 10.1111/j.1467-8624.1998.tb06195.x

Butler, E. A. (2011). Temporal interpersonal emotion systems: The "TIES" that form relationships. Personality and Social Psychology Review, 15(4), 367-393. doi: 
METHODS FOR EMOTION DYNAMICS

UNCORRECTED FIRST PROOF

$10.1177 / 1088868311411164$

Campos, J., Walle, E., Dahl, A., \& Main, A. (2011). Reconceptualizing emotion regulation. Emotion Review, 3(1), 26-35. doi: 10.1177/1754073910380975

Coco, M. I., \& Dale, R. (2014). Cross-recurrence quantification analysis of categorical and continuous time series: An R package. Frontiers in Psychology, 5, 510.

Cole, P. M., Bendezú, J. J., Ram, N., \& Chow, S.-M. (2017). Dynamical systems modeling of early childhood self-regulation. Emotion, 17(4), 684-699. doi: 10.1037/emo0000268

Cole, P. M., Lougheed, J. P., Chow, S-M., \& Ram, N. (2020). Development of emotion regulation dynamics across early childhood: A multiple time scale approach. Affective Science. doi: 10.1007/s42761-020-00004-y

Cole, P. M., Lougheed, J. P., \& Ram, N. (2018). The development of emotion regulation in early childhood: A matter of multiple time scales. In P. M. Cole \& T. Hollenstein (Eds.), Emotion Regulation: A Matter of Time (pp. 70-87). New York: Routledge.

Cole, P. M., Martin, S. E., \& Dennis, T. A. (2004). Emotion regulation as a scientific construct: Methodological challenges and directions for child development research. Child Development, 75(2), 317-333. doi: 10.1111/j.1467-8624.2004.00673.x

Feldman, R. (2007). Parent-infant synchrony: Biological foundations and developmental outcomes. Current Directions in Psychological Science, 16(6), 340-345. doi: 10.1111/j.1467-8721.2007.00532.x

Feldman, R. (2012). Bio-behavioral synchrony: A model for integrating biological and microsocial behavioral processes in the study of parenting. Parenting, 12(2-3), 154-164.

Fogel, A. (1993). Developing through relationships: Origins of communication, self, and culture. Chicago, IL: University of Chicago Press. 
METHODS FOR EMOTION DYNAMICS

UNCORRECTED FIRST PROOF

Granic, I., Hollenstein, T., Dishion, T. J., \& Patterson, G. R. (2003). Longitudinal analysis of flexibility and reorganization in early adolescence: A dynamic systems study of family interactions. Developmental Psychology, 39(3), 606-617. doi: 10.1037/00121649.39.3.606

Granic, I., O’Hara, A., Pepler, D., \& Lewis, M. D. (2007). A dynamic systems analysis of parentchild changes associated with successful "real-world" interventions for aggressive children. Journal of Abnormal Child Psychology, 35(5), 845-857. doi: 10.1007/s10802007-9133-4

Harrist, A. W., \& Waugh, R. M. (2002). Dyadic synchrony: Its structure and function in children's development. Developmental Review, 22(4), 555-592.

Helm, J. L., Miller, J. G., Kahle, S., Troxel, N. R., \& Hastings, P. D. (2018). On measuring and modeling physiological synchrony in dyads. Multivariate Behavioral Research, 53(4), 521-543. doi: 10.1080/00273171.2018.1459292

Helm, J. L., Ram, N., Cole, P. M., \& Chow, S.-M. (2016). Modeling self-regulation as a process using a multiple time-scale multiphase latent basis growth model. Structural Equation Modeling: A Multidisciplinary Journal, 23(5), 635-648. doi:

$10.1080 / 10705511.2016 .1178580$

Hollenstein, T. (2013). State space grids: Depicting dynamics across development. New York: Springer.

Hollenstein, T., Granic, I., Stoolmiller, M., \& Snyder, J. (2004). Rigidity in parent-child interactions and the development of externalizing and internalizing behavior in early childhood. Journal of Abnormal Child Psychology, 32(6), 595-607.

Hollenstein, T., Lichtwarck-Aschoff, A., \& Potworowski, G. (2013). A model of socioemotional 
METHODS FOR EMOTION DYNAMICS

UNCORRECTED FIRST PROOF

flexibility at three time scales. Emotion Review, 5(4), 397-405. doi:

$10.1177 / 1754073913484181$

Hollenstein, T., \& Tsui, T. (2019). Systems in transition: The adolescent phase transition. In S.

Kunnen, N. de Ruiter-Wilcox, M. van der Gaag, \& B. Jeronimus (Eds.), Psychosocial

Development in Adolescence: Insights from the Dynamic Systems Approach (pp. 17-31).

New York: Taylor \& Francis.

Horn, A. B., Samson, A. C., Debrot, A., \& Perrez, M. (2019). Positive humor in couples as interpersonal emotion regulation: A dyadic study in everyday life on the mediating role of psychological intimacy. Journal of Social and Personal Relationships, 36(8), 2376-2396. doi: $10.1177 / 0265407518788197$

Kenny, D. A., \& Kashy, D. A. (2011). Dyadic data analysis using multilevel modeling. New York: Routledge/Taylor \& Francis Group.

Larson, R. W., Raffaelli, M., Richards, M. H., Ham, M., \& Jewell, L. (1990). Ecology of depression in late childhood and early adolescence: A profile of daily states and activities. Journal of Abnormal Psychology, 99(1), 92-102.

Larson, R. W., \& Richards, M. H. (1994). Family emotions: Do young adolescents and their parents experience the same states? Journal of Research on Adolescence, 4(4), 567-583. doi: $10.1207 /$ s15327795jra0404_8

Lewis, M. D. (2000). Emotional self-organization at three time scales. In M. D. Lewis \& I. Granic (Eds.), Emotion, development, and self-organization: Dynamic systems approaches to emotional development (pp. 37-69). Cambridge University Press.

Lougheed, J. P. (2020). Parent-adolescent dyads as temporal interpersonal emotion systems. Journal of Research on Adolescence, 30(1), 26-40. doi: 10.1111/jora.12526 
METHODS FOR EMOTION DYNAMICS

UNCORRECTED FIRST PROOF

Lougheed, J. P. (2019). Conflict dynamics and the transformation of the parent-adolescent relationship. In S. Kunnen, M. van der Gaag, N. de Ruiter-Wilcox, \& B. Jeronimus (Eds.), Psychosocial Development in Adolescence: Insights from the Dynamic Systems Approach. New York: Routledge.

Lougheed, J. P., Benson, L., Ram, N., \& Cole, P. M. (2019). Multilevel survival analysis: Studying the timing of children's recurring behaviors. Developmental Psychology, 55(1), 53-65. doi: 10.1037/dev0000619

Lougheed, J. P., Craig, W. M., Pepler, D., Connolly, J., O’Hara, A., Granic, I., \& Hollenstein, T. (2016). Maternal and peer regulation of adolescent emotion: Associations with depressive symptoms. Journal of Abnormal Child Psychology, 44(5), 963-974. doi: 10.1007/s10802-015-0084-X

Lougheed, J. P., \& Hollenstein, T. (2016). Socioemotional flexibility in mother-daughter dyads: Riding the emotional rollercoaster across positive and negative contexts. Emotion, 16(5), 620-633. doi: 10.1037/emo0000155

Lougheed, J. P., \& Hollenstein, T. (2018). Arousal transmission and attenuation in motherdaughter dyads during adolescence. Social Development, 27(1), 19-33. doi: $10.1111 /$ sode. 12250

Lougheed, J. P., Hollenstein, T., \& Lewis, M. D. (2016). Maternal regulation of daughters' emotion during conflicts from early to mid-adolescence. Journal of Research on Adolescence, 26(3), 610-616. doi: 10.1111/jora.12211

Lougheed, J. P., Hollenstein, T., Lichtwarck-Aschoff, A., \& Granic, I. (2015). Maternal regulation of child affect in externalizing and typically-developing children. Journal of Family Psychology, 29(1), 10-19. doi: 10.1037/a0038429 
METHODS FOR EMOTION DYNAMICS

UNCORRECTED FIRST PROOF

Lougheed, J. P., Koval, P., \& Hollenstein, T. (2016). Sharing the burden: The interpersonal regulation of emotional arousal in mother-daughter dyads. Emotion, 16(1), 83-93. doi: 10.1037/emo0000105

Lunkenheimer, E. S., Olson, S. L., Hollenstein, T., Sameroff, A. J., \& Winter, C. (2011). Dyadic flexibility and positive affect in parent-child coregulation and the development of child behavior problems. Development and Psychopathology, 23(2), 577-591.

Main, A., Paxton, A., \& Dale, R. (2016). An exploratory analysis of emotion dynamics between mothers and adolescents during conflict discussions. Emotion, 16(6), 913-928. doi: $10.1037 / \mathrm{emo} 0000180$

Mancini, K. J., Luebbe, A. M., \& Bell, D. J. (2016). Valence-specific emotion transmission: Potential influences on parent-adolescent emotion coregulation. Emotion, 16(5), 567574.

Molenaar, P. C. M. (2004). A manifesto on psychology as idiographic science: Bringing the person back into scientific psychology, this time forever. Measurement: Interdisciplinary Research and Perspectives, 2(4), 201-218.

Morales, S., Ram, N., Buss, K. A., Cole, P. M., Helm, J. L., \& Chow, S.-M. (2018). Age-related changes in the dynamics of fear-related regulation in early childhood. Developmental Science, 21(5), e12633. doi: 10.1111/desc.12633

Morris, A. S., Silk, J. S., Morris, M. D. S., Steinberg, L., Aucoin, K. J., \& Keyes, A. W. (2011). The influence of mother-child emotion regulation strategies on children's expression of anger and sadness. Developmental Psychology, 47(1), 213-225. doi: 10.1037/a0021021

Nesselroade, J. R. (1991). The warp and the woof of the developmental fabric. In R. M. Downs, L. S. Liben, \& D. S. Palermo (Eds.), Visions of aesthetics, the environment, and 
METHODS FOR EMOTION DYNAMICS

UNCORRECTED FIRST PROOF

development: The legacy of Joachim F. Wohlwill (pp. 213-240). Hillsdale, N.J: Lawrence Erlbaum Associates, Inc.

Papp, L. M., Pendry, P., \& Adam, E. K. (2009). Mother-adolescent physiological synchrony in naturalistic settings: Within-family cortisol associations and moderators. Journal of Family Psychology, 23(6), 882-894. doi: 10.1037/a0017147

Ram, N., \& Diehl, M. (2015). Multiple time-scale design and analysis: Pushing towards realtime modeling of complex developmental processes. In M. Diehl, K. Hooker, \& M. J. Sliwinski (Eds.), Handbook of intraindividual variability across the lifespan (pp. 308323). New York: Routledge.

Ram, N., Gerstorf, D., Conroy, D. E., Pincus, A. L., Lorek, A., Rebar, A., ... Feldman, J. (2014). Examining the interplay of processes across multiple time-scales: Illustration with the intraindividual study of affect, health, and interpersonal behavior (iSAHIB). Research in Human Development, 11(2), 142-160. doi: 10.1080/15427609.2014.906739

Randall, A. K., \& Schoebi, D. (2015). Lean on me: Susceptibility to partner affect attenuates psychological distress over a 12-month period. Emotion, 15(2), 201-210. doi:

10.1037/emo0000043

Schoebi, D., \& Randall, A. K. (2015). Emotional dynamics in intimate relationships. Emotion Review, 1-7. doi: 10.1177/1754073915590620

Silk, J. S., Forbes, E. E., Whalen, D. J., Jakubcak, J. L., Thompson, W. K., Ryan, N. D., ... Dahl, R. E. (2011). Daily emotional dynamics in depressed youth: A cell phone ecological momentary assessment study. Journal of Experimental Child Psychology, 110(2), 241257. doi: 10.1016/j.jecp.2010.10.007

Silk, J. S., Steinberg, L., \& Morris, A. S. (2003). Adolescents' emotion regulation in daily life: 
METHODS FOR EMOTION DYNAMICS

UNCORRECTED FIRST PROOF

Links to depressive symptoms and problem behavior. Child Development, 74(6), 18691880.

Stifter, C. A., \& Rovine, M. (2015). Modeling dyadic processes using hidden Markov models: A time series approach to mother-infant interactions during infant immunization. Infant and Child Development, 298-321. doi: 10.1002/icd.1907

Stoolmiller, M. (2016). An introduction to using multivariate multilevel survival analysis to study coercive family process. In T. J. Dishion \& J. J. Snyder (Eds.), The Oxford handbook of coercive relationship dynamics (pp. 363-378). New York: Oxford University Press.

Stoolmiller, M., \& Snyder, J. (2014). Embedding multilevel survival analysis of dyadic social interaction in structural equation models: Hazard rates as both outcomes and predictors. Journal of Pediatric Psychology, 39(2), 222-232. doi: 10.1093/jpepsy/jst076

Tan, P. Z., Forbes, E. E., Dahl, R. E., Ryan, N. D., Siegle, G. J., Ladouceur, C. D., \& Silk, J. S. (2012). Emotional reactivity and regulation in anxious and nonanxious youth: A cellphone ecological momentary assessment study. Journal of Child Psychology and Psychiatry, 53(2), 197-206.

Thompson, R. A. (1994). Emotion regulation: A theme in search of definition. Monographs of the Society for Research in Child Development, 59(2-3), 25-52. doi: 10.2307/1166137

Timmons, A. C., Margolin, G., \& Saxbe, D. E. (2015). Physiological linkage in couples and its implications for individual and interpersonal functioning: A literature review. Journal of Family Psychology, 29(5), 720. doi: 10.1037/fam0000115

van der Giessen, D., Branje, S. J. T., Frijns, T., \& Meeus, W. H. J. (2013). Dyadic variability in mother-adolescent interactions: Developmental trajectories and associations with 
METHODS FOR EMOTION DYNAMICS

UNCORRECTED FIRST PROOF

psychosocial functioning. Journal of Youth and Adolescence, 42(1), 96-108. doi:

$10.1007 / \mathrm{s} 10964-012-9790-7$

Yang, X., Ram, N., Lougheed, J. P., Molenaar, P. C. M., \& Hollenstein, T. (2019). Adolescents' emotion system dynamics: Network-based analysis of physiological and emotional experience. Developmental Psychology, 55, 1982-1993. doi: 10.1037/dev0000690 